JTTEE5 19:1147

DOI: 10.1007/s11666-010-9559-z

1059-9630/\$19.00 두 ASM International

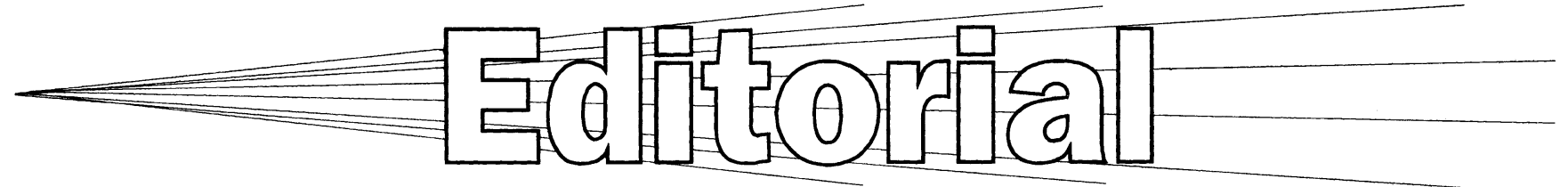

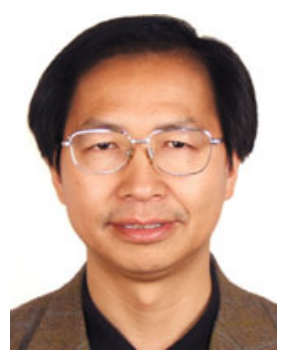

Chang-Jiu Li

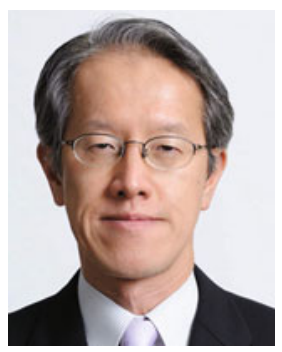

Seiji Kuroda

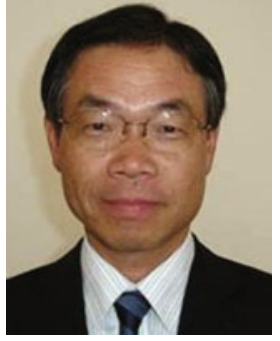

Masahiro Fukumoto

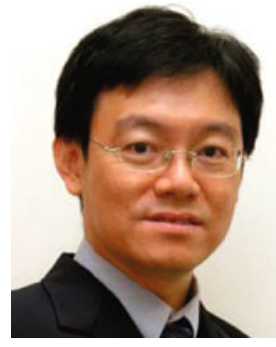

Khiam Aik Khor

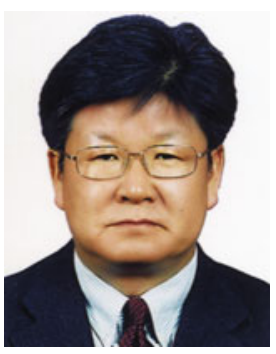

Changhee Li

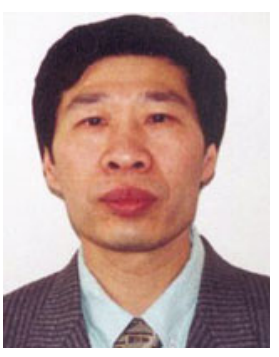

You Wang

The Asian Thermal Spray Conference (ATSC) was started in 2005. For people in Asia dedicated to the progress and applications of thermal spray, ATSC provides a forum of exchange for the latest development and achievements from industry, research institutes, and academia. For younger Asian engineers and researchers (including university students), the conference also provides great opportunities to meet with experts, learn about state-of-the-art thermal spray technology, and obtain valuable international experience. To encourage student participation, the ATSC Organizing Committee has set an affordable student registration rate and established two special awards: the Promising Young Scientist Award and the Excellent Student Award.

ATSC is held twice every 3 years in Asian countries. It is suspended only during the year that the International Thermal Spray Conference is held in Asia. The first ATSC was held in Nagoya, Japan, in 2005 with 60 papers presented. The following three conferences were held, respectively, in Korea in 2006, Singapore in 2008, and China in 2009.

During the fourth and latest ATSC held in Xi'an Jiaotong University, China, in October 22-25, 2009, a total of 145 participants shared 115 presentations, including 68 oral presentations and 50 poster presentations. Among the 145 participants, 68 were graduate students; this is in agreement with the conference's objective of encouraging young aspiring participants.

The 115 papers presented covered aspects ranging from the fundamentals that bring a deeper understanding of thermal spray science, technologies and engineering, to the latest development and applications of thermal spray coatings. Based on the recommendation of the ATSC Organizing Committee and the intentions of individual authors, 14 peer-reviewed papers among the 115 presented are published in this special issue. Even though only a small fraction of the papers presented last October are included here, the selected papers reflect the wide range of thermal spray topics presented at the conference. For example, papers on thermal spray processes cover topics ranging from plasma spraying, arc spraying, HVOF, and cold spraying to warm spraying. Papers focusing on applications include topics such as thermal barrier, corrosion protection, high temperature oxidation protection, bio-implant, photocatalyst, MEMs fabrication, etc. The papers included in this issue, in spite of their small number, reflect rather well the thermal spray activities conducted in Asia. It is sincerely hoped that this special issue of selected papers presented at the fourth ATSC will be a useful and valuable reference source for the readers.

\title{
Guest Editors
}

Chang-Jiu Li

Xi’an Jiaotong University

Xi'an, Shaanxi, China

Seiji Kuroda

National Institute of Materials Science

Tsukuba, Ibaraki, Japan

Masahiro Fukumoto

Toyohashi University of Technology

Toyohashi, Aichi, Japan

Khiam Aik Khor

Nanyang Technological University

Singapore

Changhee Li

Hanyang University

Seoul, Korea

You Wang

Harbin Institute of Technology

Harbin, Heilongjiang, China 